\title{
DAMAGE RISK ASSESSMENT OF PAINTING ON THE WOOD CONSTRUCTION IN TYPICAL TAIWANESE TEMPLE BY HOT AND HUMID CLIMATE
}

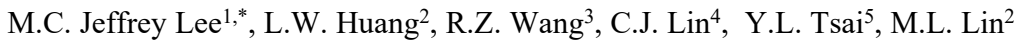 \\ ${ }^{1}$ Associate Professor, Department of interior design, National Taichung University of Science and Technology, Taichung, Taiwan. \\ ${ }^{2}$ Research Assistant, Department of interior design, National Taichung University of Science and Technology, Taichung, Taiwan. \\ - mcjl@nutc.edu.tw \\ ${ }^{3}$ Research Fellow, National Center for Research on Earthquake Engineering, Taipei, Taiwan - rzwang@ncree.narl.org.tw \\ ${ }^{4}$ Research Fellow, Taiwan Forestry Research Institute, Taipei, Taiwan - lzr5@ulive.pccu.edu.tw \\ ${ }^{5}$ Associate Research Fellow, Bureau of Culture Heritage, Ministry of Culture - ch0215@boch.gov.tw
}

Commission II, WG II/8

KEY WORDS: painting damage, wood construction, Taiwanese historical temple, indoor climate, risk assessment

\begin{abstract}
:
Most paintings were painted on the wood structure members for religion meaning and education to the prayers in the typical Taiwanese Temples. The damage of painting was induced by the micro-climate especially in the Relative humidity and Temperature various. Most typical Taiwanese temples were built by wood and brick structure with 1 open façade (semi-open space), outdoor air with different condition directly flows into indoor space to impact the material of paintings or structure members in subtropical Taiwan. When the materials absorb much damp or dry air, the volume will be expanded or shrink to damage the materials of painting layer or the basement layer. This study focuses on the damage risk assessment of painting on the wood structure members in the semi-open wooden historic temple. The test samples with painting were made by the cedar in the typical built method with wood protect oil, linen cloth, lime mortar, pigment, and surface protect oil. The test samples were put into the controlled chamber with temperature and relative humidity to test the peel off area by CNS 10757 to set up the safe range in different climate condition. The test results provide the risk assessment range in relative humidity with $55 \% \pm 15 \%$ and in temperature with $25^{\circ} \mathrm{C} \pm 10^{\circ} \mathrm{C}$ for safety range. The dangerous range may set $\pm 10 \%$ and $\pm 5^{\circ} \mathrm{C}$ from the safety range, and the very dangerous range should be destroyed the material from the boundary of EMC (Equilibrium Moisture Content), and phase change of materials. The risk assessment by benchmark in the temperature and relative humidity for easy announcing the managers to immediately trouble shooting.
\end{abstract}

\section{INTROSUCTION}

Most paintings were painted on the wood structure members for religion meaning and education to the prayers in the typical Taiwanese Temples. The paintings were painted by some famous painters on that period, they also induced art value in the historical temples. But the paintings could not move out from the constructor for keeping, they have to bear the impacts by the climate, as shown in Figure 1. Volker Schaible (Schaible, 1989) pointed out the painting damage was induced by the micro-climate, he showed the painting damage via the Relative humidity (RH) various from $80 \%$ to $30 \%$ in 36 hours. $\mathrm{Li}(\mathrm{Li}$, 2009) presented the temperature and relative humidity were the main factors to impact the painting damages in Taiwan. The results presented the best keeping method is monitoring the micro-climate and make it in less climate change for preservation of the paintings.
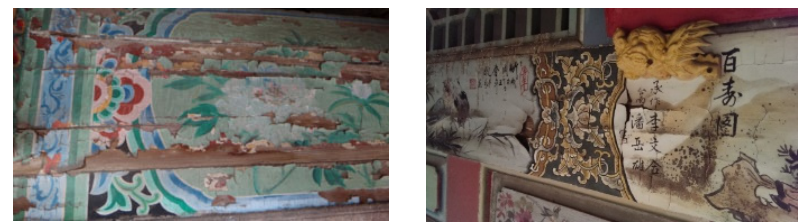

Figure 1. Painting was damaged by micro-climate change

Most typical Taiwanese temples were built by wood and brick structure with 1 open façade (semi-open space), outdoor air with different condition (Summer: $30^{\circ} \mathrm{C} \& 70 \%$, Winter: $20^{\circ} \mathrm{C}$ $\& 50 \%$ ) directly flows into indoor space to impact the material of paintings or structure members in subtropical Taiwan. The wind usually impacts the lower level because of the wind turbulence in semi-open temple as shown in Figure 2 (Lee, 2015). But it usually stops in the air tie area to accumulate the damp in the nearby materials. When the materials absorb much damp or immediately dry, the volume will be expanded or shrink to damage the materials of painting layer or the basement layer. The moisture in the air is usually involved by relative humidity and temperature (Temp) various. When the solar heat radiates into the roof, the equivalent temperature will rise up to $50^{\circ} \mathrm{C}$ to heat and expand the air volume to absorb much outdoor flowing damp into the indoor. If the temperature immediately drops down, the materials will suddenly dry and shrink due to the material unable quickly reaching the Equilibrium Moisture Content (EMC). This phenomenon causes the painting damage on the wood structure members.

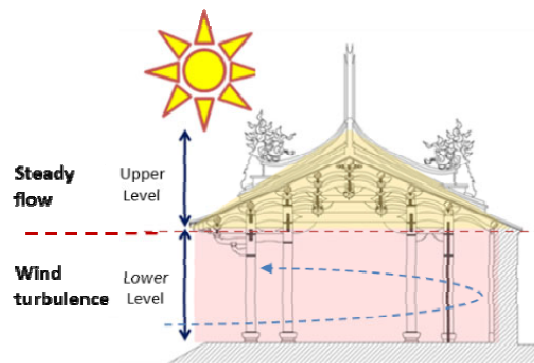




\section{Figure 2. Outdoor wet air flowing into Taiwanese Temple}

It is difficult to install any equipment to control the microclimate, because of the cultural heritage protection code in Taiwan forbidden to change the outlook of exist status. Monitoring the indoor climate various by wireless small sensors to assess the damage risk is the effective way for easy announcing the managers to immediately trouble shooting. But the critical or boundary value is the main point for warring in different climate and materials. This study focuses on the damage risk assessment of painting on the wood structure members in the semi-open wooden historic temple. The test samples with painting were made by the cedar in the typical built method with wood protect oil, linen cloth, lime mortar, pigment, and surface protect oil. The test samples were put into the controlled chamber with temperature and relative humidity to test the peel off area by CNS 10757 to set up the safe range in different climate condition.

\section{METHODOLOGY}

There are several layers with complex materials cover the wood structure members for protecting the wood and the painting. "Cedar" is most popular wood in the Taiwanese historic temple, the damage is easy impacted by inner moisture, RH, and Temp (Pan, 2005). The "Coated Basement Materials" is rather complex with different ingredients such as boiled Chinese wood oil for wood protection, linen cloth for flexible, and lime mortar for fire protection and easy painting, etc. It spends one month to complete whole process from mortar mixture, construct, cure, and complete dry (Pan, 2005). "Painting" on the basement with the typical pigment, the pigment usually mixtures with boiled Chinese wood oil and mineral powder. If the paint materials are not uniform, they would be influenced by the moisture and also crack or peel off after drying (Pan, 2005), as show in Figure 3. The painting may damage by ambient (climate) and inside (wood crack) conditions, so the experiment should be operated to check the impact factors.

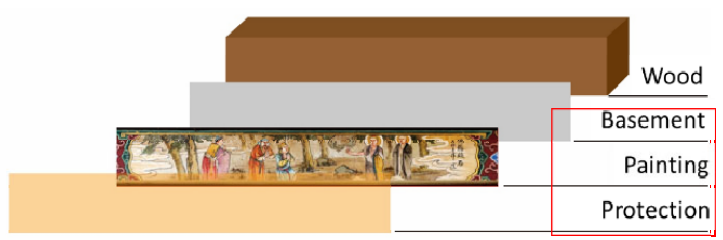

Figure 3. Different layer of the painting on the wood

Compare with a painting damage in 2010 (Hsu, 2011) and 2016 in an over 300 years Taiwanese historical temple, Beigang Chao-Tian Temple, it located at high risk climate area (Lee, 2015), as shown in Figure 4. This painting damage is located on the wooded beam in the bottom of upper level in the right building, and set a climate sensor on the beam. After long term indoor climate monitoring, the tendency shows the sudden $\mathrm{RH}$ drop from $85 \%$ to $30 \%$ and rises back to $80 \%$ in the short time. The quickly RH various causes the fatigue of the material, because of dry and wet change in the short time, as shown in Figure 5, the results are similar with the test results of Volker Schaible (Schaible, 1989).

Based on the test procedure of Volker Schaible, Psychometric Chart (PC) (Carrier Co., 1975), EMC chart of cedar (Wang, 1993), and the long term indoor climate monitoring tendency by this study to settle down the relative humidity various from $90 \%$ to $30 \%$ and the temperature various from $50^{\circ} \mathrm{C}$ to $10^{\circ} \mathrm{C}$ to assess the damage risk of the painting and base materials, as shown in Figure 6.
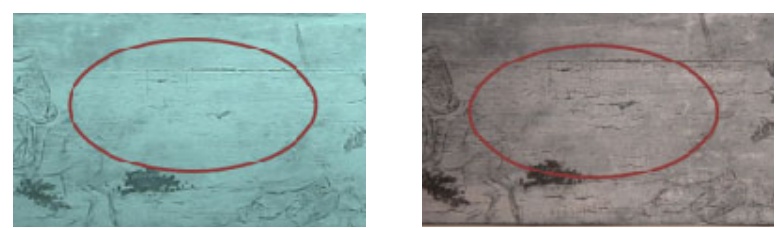

Figure 4. Painting damage between 2010 and 2016

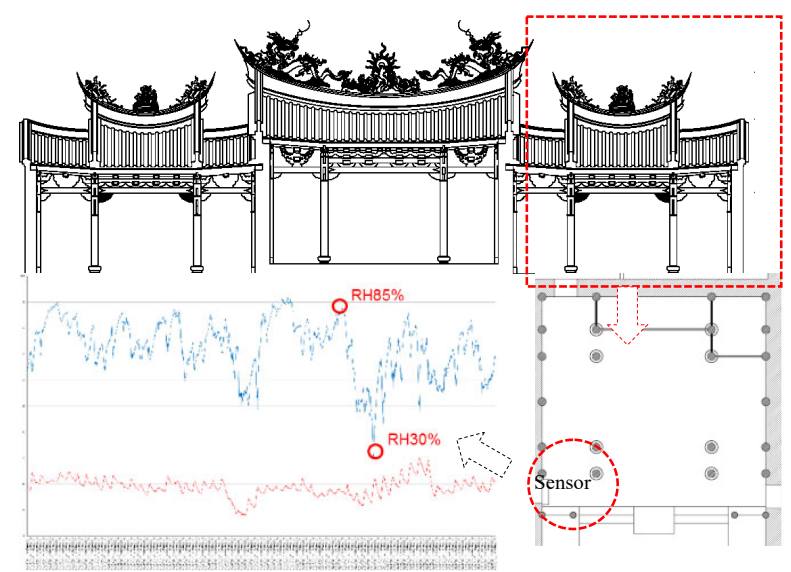

Figure 5. RH drops immediately in a historical temple
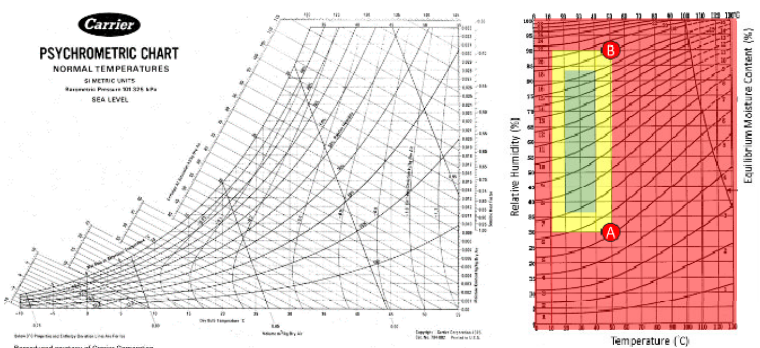

Figure 6. Test range was considered by PC, EMC chart of cedar and the monitoring tendency

\section{EXPERIMENT}

The test samples with painting were made on the cedar, they also covered several layers in the typical method (Figure 7). The first test is to observe the changes of the same test sample. Put the test sample into the Temp and RH controlled chamber (Figure 8), fix the Temp in $22^{\circ} \mathrm{C} \pm 2{ }^{\circ} \mathrm{C}$ and adjust the $\mathrm{RH}$ at $90 \%$ and at $30 \%$, the wood knot of the test sample is moving, as Figure 9.

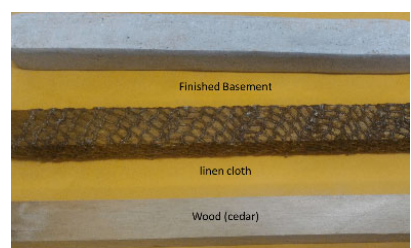

Figure 7. Test samples

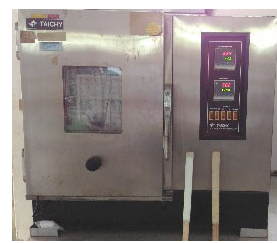

Figure 8. Chamber
The results show that the knot absorbs the damp in high $\mathrm{RH}$, the wood will go into softer status and expand the size. When the $\mathrm{RH}$ is dropping in the short time, the damp will vapour and dried the knot to go into harder status and shrink the size. Due to the knot size is changed, the knot position is also change a little bit, as shown in Figure 9. As the same result, the painting 
is also changed the original structure to be the damage. The other impact is painting stays in the softer status, it is easily damaged by extra forces, such as wind flow, wind driven rain, etc.
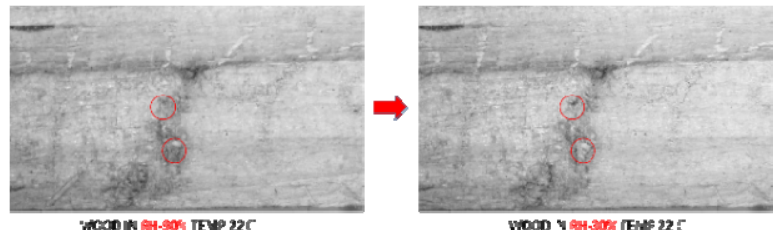

Figure 9. Wood knot was affected by RH various

The second test is followed CNS 10757 (China National Standard, CNS, 1995) to compare the painting peel off conditions of the same test sample in high Temp and different RH. Also put the test sample into the chamber, fix the Temp in $50{ }^{\circ} \mathrm{C}$ and adjust the RH from $90 \%$ to $30 \%$ during 2 hours. The results show the painting on the wood could be easy peeled off at RH $90 \%$, because of lime mortar absorbing damp to lose the adhesion and friction, as shown in Figure 10. Therefore, when climate stays in high RH and high Temp, the materials drop the original physical characteristic to be easy damaged by extra forces or self-weight.

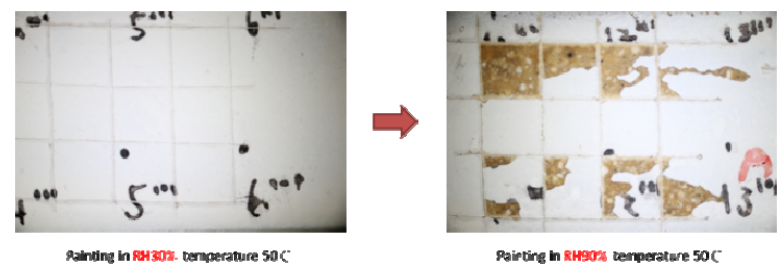

Figure 10. Painting peel off status in different $\mathrm{RH}$ in $50^{\circ} \mathrm{C}$

\section{DISCUSSION}

Cracks did not appear on the samples in a short period with $\mathrm{RH}$ various, except the surface expansion and contraction. When paintings stay in high Temp and RH condition, the peel off rate reaches to $50 \%$. The adhesive force and friction of painting materials belong in fragile status while high Temp and RH. But the paintings stay in low Temp and $\mathrm{RH}$, the material would be damaged quickly by shrinkage and cracks. To submit a range of risk assessment is more flexible, as shown in Figure 11. The peel off percentage in different relative humidity and temperature. If the peel off percentage in $2 \%$ is acceptable, the damage risk in relative humidity could be defined between $40 \%-70 \%$, and the damage risk in temperature could be set between $15-30^{\circ} \mathrm{C}$.

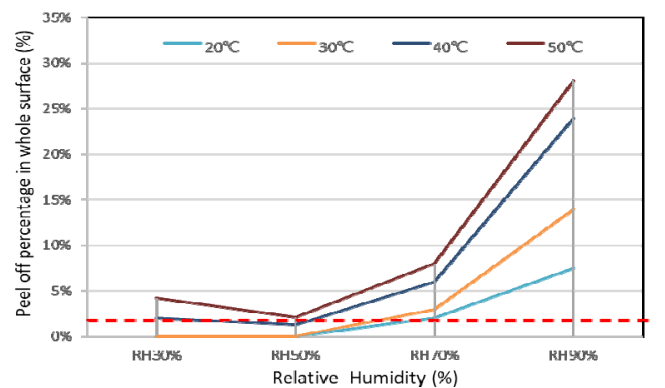

Figure 11. Painting peel percentage in different condition

As we know the damp is the key point for physical damage of painting materials, and many recommends keep around $50 \% \pm$ $5 \%$ (ASHRAE 2003), $45 \% \pm 8 \%$ and $45 \% \pm 18 \%$ (Mecklenburg,
2012) in different conservation climate. Based on the test results, EMC chart of cedar and past study in the monitoring tendency (Lee et al, 2015). This study submits a risk assessment range by benchmark in the Temp and $\mathrm{RH}$ for preservation of painting on the wooden historic temple in hot and humid climate Taiwan. The outdoor RH stays around $60-70 \%$ and outdoor climate directly impacts indoor climate due to the semiopen style in the most historic temples. For the long term EMC balance, the material could bear higher RH but not bear lower RH comparing with the dry area. In the other hand, the Temp range is around $20-30^{\circ} \mathrm{C}$ without considering the equivalent temperature. Refer with the Psychometric Chart to know the $\mathrm{RH}$ is directly related with Temp, especially in Wet Ball Temp. Therefore, the risk assessment ranges for semi-open building set in RH with $55 \pm 15 \%$ and in Temp with $25^{\circ} \mathrm{C} \pm 10^{\circ} \mathrm{C}$ for safety range. The dangerous range may set $\pm 10 \%$ and $\pm 5^{\circ} \mathrm{C}$ from the safety range. The very dangerous range should be destroyed the material from the boundary of EMC, and phase change of materials, as shown in Figure 12.
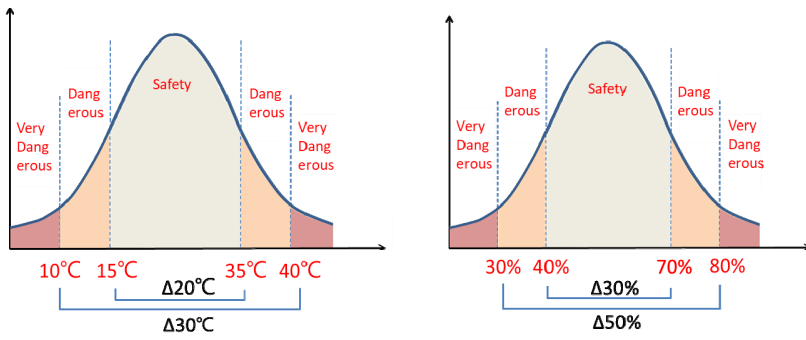

Figure 12. Risk assessment in hot and humidity area

\section{CONCLUSION}

Preservation of Painting damage on the wood structure members in the Taiwanese historic temple is not easy due to the semi-open building. The indoor climate usually impacted by outdoor climate via wind flow. Submit a risk assessment by benchmark in the Temp and RH for easy announcing the managers to immediately trouble shooting.

\section{ACKNOWLEDGEMENTS}

The author will thank Bureau of Cultural Heritage, Ministry of Culture Taiwan to support finance and technic. We also thank for Taiwan Forestry Research Institute and National Centre for Research on Earthquake Engineering to support the technic and experimental instrument.

\section{REFERENCES}

ASHRAE Standard 21.13, 2003. ASHRAE, USA

Carrier Co. Ltd., 1975. "Psychometric Chart”, Carrier Co. Ltd., USA

China National Standard, CNS \#10757, 1995. General Painting performance check standard, Bureau of Standards, Metrology and Inspection, Taiwan

Hsu M.F., 2011. Investigation and conservation plan of painting damage on the wooden beam in National Monument (Beigang Chao-Tian Temple), Yunlin County Government, ISBN: 9860280452, 9789860280456 
Lee, M.C., Tsai, Y.L., Lin, M.L., Huang, L.W., Chen C.Y., 2015. Monitoring Mechanism in Preservation of Monuments in Hot and Wet Climate Area, $25^{\text {th }}$ International CIPA Symposium, DOI:10.5194/isprsarchives-XL-5-W7-271-2015

Lee M.C., Lin M.L., 2015. First stage planning of Preservation Monitoring of Historic Monuments, Project Report, Institute of Cultural Heritage Preservation Research, Bureau of Cultural Heritage, Ministry of Culture, Taiwan

Li Jhih-Shang, 2009. The study of deteriorated conditions of polychrome wooden objects in the religion buildings in Taiwan, Master Thesis of Tainan National University of the Arts.

Mecklenburg, M.F., Tumosa, C.S., and Erhardt, D., 1998, 'Structural response of painted wood surfaces to changes in ambient relative humidity', in Painted Wood: History and Conservation, ed. V. Dorge and F.C. Howlett, The Getty Conservation Institute, Los Angeles, pp.464-483.

Pan Hsi, 2011. The Study on the Basement Materials Coated for Architectural paints - the contemporary ones in Taiwan as an Example, Master thesis, of Tainan National University of the Arts

Wang S.Y., 1993. Wood Physical, Taiwan, ISBN: 9789862958575.

Schaible Volker, 1989. Der film “ Vom Ateme der Bilder"

Revised June 2019 